\title{
Laser-Assisted Generation and Detection of Non-Nuclear Low-Energy Neutrino-Antineutrino Beams
}

\author{
Jeff W. Eerkens \\ PO Box 8566, Woodland, CA, USA \\ Email: eerkensj@missouri.edu,neutreklaser@gmail.com
}

How to cite this paper: Eerkens, J.W. (2018) Laser-Assisted Generation and Detection of Non-Nuclear Low-Energy Neutrino-Antineutrino Beams. Journal of Modern Physics, 9, 1291-1296.

https://doi.org/10.4236/jmp.2018.96077

Received: March 28, 2018

Accepted: May 22, 2018

Published: May 25, 2018

Copyright $\odot 2018$ by author and Scientific Research Publishing Inc. This work is licensed under the Creative Commons Attribution International License (CC BY 4.0).

http://creativecommons.org/licenses/by/4.0/

(c) (i) Open Access

\begin{abstract}
It is argued that conservation of energy, momentum, and spin, and QM transition probabilities, allow the generation and detection of low-energy $(\mathrm{eV})$ neutrinos and antineutrinos in intra-atomic (non-nuclear) laser transitions. Two-quantum transitions between an upper and lower excited state in a lasing medium can support paired emissions of a neutrino and a recoiling counter-propagating anti-neutrino, each carrying half of the lasing transition energy. Their propagations are in opposite directions along the same axis as that of the intracavity laser beam in the lasing medium. Estimates show that the probability of this two-quantum event is on the order of $10^{-7}$ compared to a one-quantum stimulated emission of a laser photon. Absorptions or emissions of single antineutrinos or neutrinos by molecular/atomic matter are impossible because they carry spins $\mathrm{s}= \pm \mathrm{h} / 2$ which violates $\Delta \mathrm{s}= \pm \mathrm{nh}(\mathrm{n}=\mathrm{in}-$ teger) required for such processes. However, inside a laser, emissions of photons from excited states can be stimulated by neutrinos or anti-neutrinos passing through, provided their undulation frequency is resonant with the transition frequency. This is because in stimulated emissions, neutrinos or antineutrinos are not absorbed, and spin conservation violation is not an issue. Thus, detection of the passage of a laser-generated antineutrino or neutrino beam through a second "receiver" laser is possible provided that the transition energy in the second laser equals half the transition energy of the laser that emits the antineutrino-neutrino beam to be detected.
\end{abstract}

\section{Keywords}

Non-Nuclear Neutrino Generation and Detection, Neutrino

Communications, Lasers 


\section{Introduction}

In the 1930s, to explain intra-nuclear beta-decays, Enrico Fermi postulated the existence of a then-unobservable neutrino to accommodate spin, energy, and momentum conservation of the emitted betas ( $=\mathrm{MeV}$ electrons). The conservation laws of momentum and energy forced him to that conclusion in order to justify his observation of beta-emissions with a continuous (not discrete) energy spectrum. Today, the existence of neutrinos is well established, but experiments have been limited to studies of intra-nuclei-born neutrinos and antineutrinos with $\mathrm{MeV}$ energies that can induce observable inverse-beta-decay transitions in nuclei. Neutrinos and antineutrinos with $\mathrm{eV}$ energies have not been studied, because heretofore there was no way to observe their presence. Nevertheless, Fermi's observations do prove the existence of $\mathrm{eV}$ neutrinos and antineutrinos in the energy domain of lasers and masers.

With the assurance that $\mathrm{eV}$ neutrinos (henceforth the word "neutrinos" includes antineutrinos) do exist, an investigation of producing intra-atomic generated $\mathrm{eV}$ neutrinos was undertaken. In the late 1970s, some exploratory experiments with Nd lasers were carried out and a patent was obtained [1]. The experiments neither proved nor disproved the concept because of potential noise/interference signals that were registered by the detector [2]. At the time, other interesting laser developments were underway, and a return to further explore intra-atomic or intra-molecular $\mathrm{eV}$ neutrino generation and detection concept did not occur until 2012. By that time, it was realized that a connection with the old neutrino theory of photons [2] [3] was unnecessary, and a successful demonstration was more likely if the detection laser was operating at a laser wavelength twice that of the emitter. This latter conclusion followed from a more thorough review of second-order two-quantum atomic transition elements and resonant maxima, in addition to careful observance of conservation laws (momentum, energy, and spin).

\section{Improved Theory of Laser-Assisted Neutrino Detection}

The basic postulate is that in a lasing medium, instead of a photon stimulating the emission of a second like (copied) photon, it is possible that occasionally the stimulating photon generates the emission of a neutrino paired with a recoiling antineutrino with equal energy. These propagate in opposite directions along the same center line as the intracavity laser beam. But whereas the intracavity laser beam is amplified by photon-multiplying stimulated emissions and reflections from end mirrors, the generated neutrino beam passes directly through the end mirrors (because $s= \pm h / 2$, see below). The probability for an incoming photon to generate a recoiling neutrino-antineutrino pair in the stimulated de-excitation of an excited atom molecule, is on the order of $10^{-7}$ compared to the probability that an incoming laser photon stimulates emission of a second laser photon with the same frequency thereby causing laser multiplication. Such a weak second-order effect is typical of two-quantum changes compared to a one-quantum change in 
atomic transitions (e.g. Raman effect). For maximum neutrino beam generation, the reflectivity of the laser end mirrors should be as high as possible $(99 \%+)$ to provide high stimulated emission rates in the laser and thus high neutrino beam generation.

An improvement over the 1980 concept was the later realization that second-order (weak) stimulated emissions of two quanta can take place between two atomic/molecular energy levels. There is no need to have three- or more communicating energy levels as proposed in 1980. This means that the lasing frequency of the neutrino-beam detector/receiver must be one half of that of the emitter. It requires use of different lasers with different wavelengths for the generation versus the detection of $\mathrm{eV}$ neutrinos.

Neutrinos $(s=-h / 2)$ and antineutrinos $(s=+h / 2)$, like photons, undulate through space in spiraling waves. Except (anti)neutrinos have spins $s= \pm h / 2$ instead of spins with $s=0, \pm 1 h, \pm 2 h$, possessed by photons. When interacting with matter, an absorption or emission requires a spin change of $\Delta s= \pm 1$ to change the electronic or vibrational exited state of an atom or molecule.

Because of spins $s= \pm h / 2$, single neutrinos or antineutrinos cannot be absorbed by matter and pass straight through the earth. High-energy (MeV) neutrinos or antineutrinos also experience such free passage, but occasionally they can meet an intra-nuclear electron or positron (with spin $\pm h / 2$ ) allowing an "inverse-beta-decay" (ibd) spin-conserving absorption that is detectable.

To detect $\mathrm{MeV}$ neutrinos, enormous swimming-pool-sized detection systems filled with inverse-beta-decay (ibd)-sensitive nuclei have been constructed, surrounded by some thousand light flash sensors. Only ten or so ibd events per day are detected however from intra-nuclear passages by $\mathrm{MeV}$ neutrinos. This low rate is partly due to the small radius of nuclei (on the order of $10^{-12} \mathrm{~cm}$ ) with intercept cross-sections of $10^{-24} \mathrm{~cm}^{2}$. A “swimming-pool" detector filled with say $10^{20}$ ibd-sensitive nuclei, only observes 8.64 ibd events per day when irradiated by $10^{6}$ high-energy $(\mathrm{MeV})$ nuclear neutrinos/sec passing through a $100,000 \mathrm{~cm}^{2}$ pool wall, assuming an intra-nuclear ibd-reaction probability of $10^{-1}$ per nucleus and intercept cross-section of $10^{-24} \mathrm{~cm}^{2}$ per ibd-sensitive nucleus.

In proposals to utilize nuclear $\mathrm{MeV}$ neutrinos for through-the-earth communications, one has to solve two problems: 1) produce a modulated beam of neutrinos, and 2) detect such a beam. Neutrinos and antineutrinos are generally emitted in all spatial directions in intra-nuclear transitions. Weber describes a way in which neutrino emissions by a particle accelerator might be lined up in one direction by applying a strong DC magnetic field at the accelerator emitter so as to create a neutrino beam. This beam could then be modulated to impose and transfer information similar to that proposed by the author [2] [3] [4]. For a receiver, Weber [5] proposes to detect the passage and presence of modulated $\mathrm{MeV}$ neutrino beams via a "stiff" crystal that reacts to resonant scattering of neutrinos or antineutrinos with undulation frequencies on the order of $3 \times 10^{20}$ $\mathrm{Hz}(=1.23 \mathrm{MeV})$. Clearly, $\mathrm{MeV}$ neutrino beams so formed and detected (if technically feasible), require elaborate expensive means for implementation. 
Using $\mathrm{eV}$ neutrinos generated by intra-atomic processes instead, has many advantages. Neutrino intercept cross-sections of atoms are on the order of $10^{-16}$ $\mathrm{cm}^{2}$, which is $10^{8}$ times larger than of nuclei. Also, generation of paired counter-propagating neutrinos and antineutrinos recoiling off each other in laser-stimulated emissions, creates neutrino beams that overlap the laser beam. Such beams allow long-distance communications between emitters and receivers as described in [2] and [3].

Visualization of intra-molecular $\mathrm{eV}$ neutrino generation and detection, and a simple proof-of-principle demonstration experiment, were given by Figures 1 and 2 in [2] and [3]. In what follows we re-list pertinent expressions for theoretical rate events presented in [1] [2] [3] [4].

\section{Theoretical Rates}

As described in References 2 and 3, a neutrino communication system that utilizes intra-atomic-generated $\mathrm{eV}$ neutrinos, comprises an Emitter laser with laser wavelength $\lambda_{E}$ and a Receiver laser operating at laser wavelength $\lambda_{R}=2 \lambda_{E}$. Inside the Emitter laser, pairs of neutrinos and antineutrinos are generated in two-quantum laser-photon-stimulated emissions together with one-quantum laser photons. Both produce intracavity beams that overlap. However, while the laser photon beam is amplified by bidirectional running waves due to near- $99 \%$ reflections from laser end mirrors, the neutrino beam exits through both end mirrors without reflection because $s=h / 2$. Detection of the $\mathrm{eV}$ neutrino beam some distance away, is accomplished by the Receiver laser with wavelength $\lambda_{R}=$ $2 \lambda_{E}$ as mentioned. Using labels $v$ and $\underline{v}$ for neutrinos and antineutrinos respectively, and $\gamma$ for a photon, the following theoretical rate expressions were derived from microscopic quantum transition physics [4] [6] [7] [8], with abbreviations spon $=$ spontaneous and stem $=$ stimulated emission

1) EMITTER/SOURCE LASER

a) Spontaneous and Stimulated Emission of a Photon $(\gamma)$ from an Excited Atom or Molecule

The spontaneous Einstein emission rate of a photon by an excited atom or molecule is given by the well-known expression [6]:

$$
{ }^{\text {spon }} k_{k n}^{(\gamma)}=(8 \pi / 3)\left\{e^{2} /(\hbar c)\right\}\left\langle y_{k n}\right\rangle^{2} E_{k n}^{3} /\left(\hbar^{3} c^{2}\right)=2.379 \times 10^{7}\left\langle y_{k n}\right\rangle^{2} E_{k n}^{3}, \mathrm{~s}^{-1}
$$

Here the transition is from excited state $k$ to $n$, while $E_{k n}=E_{k}-E_{n}$, and $\left\langle y_{k n}\right\rangle$ is the Cartesian transition displacement element given by the QM relation ( $e=$ electronic charge):

$$
\left\langle y_{k n}\right\rangle=\left\langle\mu_{k n}\right\rangle / e=\int_{\Omega} \psi_{n} y \psi_{k} \mathrm{~d} \Omega
$$

with $y=$ displacement, $\mu$ dipole moment, $\Omega=$ integration space around atom where $\psi_{k}, \psi_{n}$ are non-zero, and $\psi_{k}, \psi_{n}=$ wave functions of states $k$ and $n$.

For photon-stimulated photon emissions $\gamma \rightarrow \gamma$, one finds [6]:

$$
\begin{aligned}
{ }^{s t e m} k_{k n}^{(\gamma \rightarrow \gamma)} & =\left(4 \pi^{2} / 3 \hbar\right)\left\{e^{2} /(\hbar c)\right\}\left\langle y_{k n}\right\rangle^{2} I_{L}\left(\omega_{\gamma}=\omega_{k n}\right) / \Delta \omega_{k n} \\
& =3.035 \times 10^{6}\left\langle y_{k n}\right\rangle^{2} I_{L}\left(\omega_{\gamma}=\omega_{k n}\right) / \Delta \omega_{k n}, \mathrm{~s}^{-1}
\end{aligned}
$$


In the second part of expressions (1) and (3), the units for $\left\langle y_{k n}\right\rangle$ are Angstroms, $E_{k n}$ is in $\mathrm{eV}$, while $I_{L}$ is laser power in $\mathrm{W} / \mathrm{cm}^{2}$ and resonance width $\Delta \omega_{k n}$ is in $\mathrm{cm}^{-1}$. For example for a Neodymium laser one has $\left\langle y_{k n}\right\rangle \approx 1$ Angstrom, $E_{k n} \approx 1 \mathrm{eV}, I_{L} \approx 1 \mathrm{~W} / \mathrm{cm}^{2}$, and $\Delta \omega_{k n} \approx 1 \mathrm{~cm}^{-1}$.

b) Spontaneous and Stimulated Neutrino Pair ( $v \underline{v})$ Emission from Excited

\section{Atom/Molecule}

For spontaneous second-order two-quanta emission rates and photon-stimulated emission rates of $\underline{v} \underline{v}$ pairs by an excited atom or molecule transitioning from excited state $\mathrm{k}$ to $\mathrm{n}$, one finds, using Feynman's QM formulation for two-quanta transitions [4] [7] [8]:

$$
\begin{aligned}
{ }^{s p o n} k_{k n}^{(v \underline{v})} & =\left(2^{7} \pi^{2} / 3\right)\left[\left|\mu_{k n}\right| /(\hbar c)\right]^{4}\left[E_{k n}^{5} /\left(\hbar^{3} c^{2}\right)\right] \\
& =\left(2^{7} \pi^{2} / 3\right)\left[e^{2} /(\hbar c)\right]^{2}\left\langle y_{k n}\right\rangle^{4}\left[E_{k n}^{5} /\left(\hbar^{5} c^{4}\right)\right] \\
& =2.236\left\langle y_{k n}\right\rangle^{4} E_{k n}^{5}, \mathrm{~s}^{-1} \\
{ }^{\text {stem }} k_{k n}^{(\gamma \rightarrow v \underline{v})}= & \left(2^{6} \pi^{3} e^{4} /\left(3 \hbar^{5} c^{4}\right)\right)\left\langle y_{k n}\right\rangle^{4} E_{k n}^{2} I_{L}\left(\omega_{\gamma}=\omega_{k n}\right) / \Delta \omega_{k n} \\
= & 0.2853\left\langle y_{k n}\right\rangle^{4} E_{k n}^{2} I_{L}\left(\omega_{\gamma}=\omega_{k n}\right) / \Delta \omega_{k n}, \mathrm{~s}^{-1}
\end{aligned}
$$

Again, units in the last parts of (4) and (5) are Angstroms for $\left\langle y_{k n}\right\rangle, \mathrm{eV}$ for $E_{k n}$, Watts $/ \mathrm{cm}^{2}$ for $I_{L}$, and $\mathrm{cm}^{-1}$ for the resonance width $\Delta \omega_{k n}$. (Note also that $e^{2} / \hbar c=1 / 137$ is used.)

Comparing second-order $v v$ pair emission rates to first-order photon $(\gamma)$ emission rates for spontaneous and stimulated cases, one finds that ratios are the same for both:

c) Ratio $(\gamma \rightarrow v \underline{v}) /(\gamma \rightarrow \gamma)$ for Stimulated Emissions and for Spontaneous Emissions:

$$
{ }^{s t e m} k_{k n}^{(\gamma \rightarrow r \underline{v})} /{ }^{s t e m} k_{k n}^{(\gamma \rightarrow \gamma)}={ }^{s p o n} k_{k n}^{(v \underline{v})} /{ }^{s p o n} k_{k n}^{(\gamma)}=0.940 \times 10^{-7} E_{k n}^{2}\left\langle y_{k n}\right\rangle^{2}
$$

where $E_{k n}$ is in eV units and $\left\langle y_{k n}\right\rangle$ is in Angstroms again.

\section{2) RECEIVER/DETECTION LASER}

The rate at which frequency-resonant neutrinos (or antineutrinos) can stimulate the emission of photons from an excited atom or molecule is proportional to the neutrino flux and equals:

d) Stimulated Photon $(\gamma)$ Emission by Resonant Fly-through Neutrinos ( $v$ or $\underline{v})$ :

$$
\begin{aligned}
{ }^{\text {stem }} k_{k n}^{(v \rightarrow \gamma)} & =\left[\left(4 \pi^{2} / 3 \hbar\right) e^{2} /(\hbar c)\right]\left\langle y_{k n}\right\rangle^{2} E_{k n} \varphi_{v}\left(\omega_{v}=\omega_{k n}\right) / \Delta \omega_{k n} \\
& =4.862 \times 10^{-13}\left\langle y_{k n}\right\rangle^{2} E_{k n} \varphi_{v}\left(\omega_{v}=\omega_{k n}\right) / \Delta \omega_{k n}, \mathrm{~s}^{-1}
\end{aligned}
$$

Here $\varphi_{v}$ is the neutrino flux in $\mathrm{cm}^{-2} \cdot \mathrm{s}^{-1}$, and as before, the units for $\left\langle y_{k n}\right\rangle$ are Angstroms, $E_{k n}$ is in $\mathrm{eV}$, while the resonance width $\Delta \omega_{k n}$ is in $\mathrm{cm}^{-1}$ in the second part of expression (7).

e) The ratio of neutrino $v \rightarrow \gamma$ versus photon $\gamma \rightarrow \gamma$ stimulated emission rates is: 


$$
{ }^{s t e m} k_{k n}^{(v \rightarrow \gamma)} /{ }^{s t e m} k_{k n}^{(\gamma \rightarrow \gamma)}=\varphi_{v}\left(\omega_{v}=\omega_{k n}\right) / \varphi_{\gamma}\left(\omega_{\gamma}=\omega_{k n}\right)
$$

The result (8) shows that resonant neutrino-stimulated emission rates are like resonant photon-stimulated emission rates and controlled by incident flux levels. In both these stimulated photon-emission cases, there is no spin exchange. Only frequency resonance is required.

\section{Note}

A proposal to examine $\mathrm{eV}$ neutrino generation and detection concepts according to the improved theory was first announced in 2013. Subsequently, an experimental test program was prepared as described in [2] and [3], requiring a high-power laser for the emitter. Such a laser was available from another project and the plan was to "piggy-back" the neutrino tests in that program. Unfortunately, funding for the other project was repeatedly delayed which derailed the plans to verify laser-assisted neutrino-generation. Financial assistance to complete the demonstration project is welcome (please contact the author). As far as is known, no one has disproved the concept to generate and detect intra-atomic laser-generated eV neutrinos.

\section{References}

[1] Eerkens, J.W. (1980) Neutrino Communication Arrangement. US Patent 3430.046.

[2] Eerkens, J.W. (2013) Applied Physics B: Lasers and Optics, 111, 323-331.

[3] Eerkens, J.W. (2013) Proceedings of SPIE, 8832, Paper 1A.

[4] Eerkens, J.W. (2010) Semi-Classical Calculation of Neutrino Emission and Stimulation Probabilities. Prodev Consultants unpublished Paper.

[5] Weber, J. (1994) Method and Apparatus for Generating Neutrinos and/or Antineutrinos, a Neutrino Modulation Method, and a Neutrino Beam Generator. US Patent 5276717.

[6] Eerkens, J.W. (1973) Model Equations for Photon Emission Rates and Absorption Cross-Sections; Vol II Rocket Radiation Handbook. US Air Force FTD, No CW-01-01-74; CDC No AD-A007946, Dec (USAF Contract F33657-72-C-0850).

[7] Feynman, R.P. (1961) Quantum Electrodynamics. W.A. Benjamin, Inc, New York.

[8] Cohan, N.V. and Hameka, H.F. (1966) Physical Review Letters, 16, 478. 